Résumés des conférences et travaux

\title{
Histoire et philologie de la Mésopotamie
}

\section{Michaël Guichard}

\section{(2) OpenEdition Journals}

Édition électronique

URL : https://journals.openedition.org/ashp/1921

DOI : $10.4000 /$ ashp. 1921

ISSN : 1969-6310

Éditeur

Publications de l'École Pratique des Hautes Études

\section{Édition imprimée}

Date de publication : 1 septembre 2017

Pagination : 17-22

ISSN : 0766-0677

\section{Référence électronique}

Michaël Guichard, « Histoire et philologie de la Mésopotamie », Annuaire de l'École pratique des hautes études (EPHE), Section des sciences historiques et philologiques [En ligne], 148 | 2017, mis en ligne le 21 septembre 2017, consulté le 06 juillet 2021. URL : http://journals.openedition.org/ashp/1921 ; DOI : https://doi.org/10.4000/ashp.1921 


\title{
HISTOIRE ET PHILOLOGIE DE LA MÉSOPOTAMIE
}

\author{
Directeur d'études : M. Michaël Guichard
}

Programme de l'année 2015-2016 : Guerre et paix en Haute Mésopotamie. Lecture et analyse de la documentation paléo-babylonienne issue de Nahur et de ses environs (région du Haut Habur).

Le séminaire de cette année s'est penché sur le cas d'une capitale régionale, Nahur, une cité située dans le Triangle du Habur. Sa localisation précise est inconnue même si plusieurs tells ont déjà été proposés comme candidats possibles. Nous pouvons seulement dire qu'elle se trouvait dans le Nord-Ouest du Triangle du Habur non loin de la cité d'Urkiš identifiée à Tell Mozan, dans la zone frontalière syro-turque.

Nahur est abondamment citée dans les archives de Mari du XVIII ${ }^{\mathrm{e}}$ siècle mais assez peu au-delà de Mari. La brusque augmentation de données la concernant tient en partie au fait que son roi Zimrī-Lim l'a annexée et y a installé une petite garnison militaire. L'instabilité chronique des Marches septentrionales de Mari où se situait Nahur donna lieu à un abondant courrier que nous avons eu la chance de retrouver presque intact dans le palais de Zimrī-Lim. L'agent le plus prolixe et le mieux renseigné était le gouverneur de Nahur qui durant 8 années expédia vers la capitale du Moyen-Euphrate rapport sur rapport.

Nous avons suivi quelques étapes du processus qui a conduit le roi de Mari à conquérir la ville et à l'administrer lui-même, puis avons examiné la phase d'installation du gouverneur et la restauration de l'économie palatiale, la ville étant passablement ruinée. Nous nous sommes appuyés pour cela sur un ensemble de textes de comptabilité qui permettent notamment de fixer quelques repaires chronologiques précis, puis sur une sélection de lettres allant de l'avènement de Zimrī-Lim jusqu'au milieu de son règne de 13 ans.

Pourtant avant d'entrer dans les détails complexes d'une histoire plutôt bien documentée, j'ai tenu à resituer la prise de cette capitale de Haute Mésopotamie par un roi du Moyen-Euphrate, un événement certes local, dans le temps long de la Haute Djéziré. La prise de la ville n'a pas été seulement une conquête militaire, elle a surtout l'intérêt d'avoir été une entreprise de colonisation qui a nécessité la coopération d'une partie des populations environnantes. L'épisode offre ainsi l'opportunité d'étudier en profondeur la société de la Haute Djéziré, son économie et ses institutions. Au préalable, l'histoire du Haut-Habur a été retracée, en donnant la priorité à l'information textuelle, depuis le temps des archives d'Ebla jusqu'à l'époque des royaumes amorrites au début de la Première Dynastie de Babylone, en passant par la fondation de l'empire d'Akkad, l'intermède des Goutis, le siècle des rois de la Troisième Dynastie d'Ur, les « invasions amorrites » et l'émergence des petits royaumes amorrites de la Haute Djéziré. Malheureusement les sources écrites concernant la région du Habur sont jusqu'aux archives paléo-babyloniennes de Mari très inégales, pour ne 
pas dire pauvres, sans compter une chronologie générale encore incertaine en raison en particulier de notre méconnaissance de la période dite des Goutis (soit le laps de temps écoulé entre les règnes de Šar-kali-šarrī d'Akkad et d'Utu-hegal d'Uruk). Malgré tout, des éléments de continuité et de rupture peuvent être mis en évidence.

La Haute Djéziré connaît dans la première moitié du troisième millénaire une phase de développement et de renouveau urbain. De grandes villes du type Kranzhügel ( " ville en couronne » ou encore « Cup and Sauser ») se sont formées. Tell Khuera (dans la zone du Balikh) est le modèle le plus impressionnant de cette série. Des bâtiments monumentaux sont là pour témoigner de la centralisation du pouvoir; 1'architecture des palais et des temples est ostentatoire. Les édifices dégagés à Nagar sont les plus massifs : des bâtiments officiels, une cour et un temple forment un seul complexe. Si Nagar est la capitale du Haut-Habur, c'est surtout Tell Beydar (sur le wadi Awaiğ) un de ses satellites (situé à une quarantaine de km de Nagar et une vingtaine de kms au sud d'Urkiš) qui illustre le mieux ce phénomène puisque son niveau archéologique contemporain d'Ebla et Mari présargoniques était intégralement préservé. L'acropole était couronnée d'un palais et d'un temple. Au nord du palais se trouvaient des bergeries isolées du reste de la ville. Au sud du temple se pressaient trois autres ce qui rappelle le quartier « religieux » contemporain de Mari.

La forte densité d'occupation ne sera égalée qu'à l'époque néo-assyrienne. De compacts quartiers d'habitations se forment dans les villes où des familles étendues cohabitent. Les sites s'étendent dans tout le Triangle du Habur jusque dans le nord du Djebel Abd-el-Aziz. La région est d'ailleurs propice à l'agriculture, ses cours d'eau sont encore bordés de forêts galeries, l'élevage y joue un rôle important et ses équidés (BAR.AN venus d'Iran) sont, par exemple, réputés à Ebla. Des vétérinaires spécialement chargés des soins de ces luxueuses montures se rendent là-bas depuis Nagar.

Si l'usage de tablettes à chiffres est ancien en Djéziré, attestant d'une administration même primitive, les premiers textes eux ont été trouvés à Mozan, Brak et surtout Beydar autour de 2400. Les textes administratifs indiquent que Nabada (le nom antique du site) était typique d'une cité-État qui employait une large partie de la population. Celle-ci n'était qu'un satellite de Nagar qui contrôlait au moins une vingtaine d'autres centres de la région. La langue des textes est l'akkadien ce qui témoigne de la forte influence de Kiš. L'onomastique et la toponymie locales étaient majoritairement sémitiques.

Vers 2400-2350, Nagar participe intensivement à la vie politique qui relie Kiš à l'est, Ebla à l'ouest et Mari au sud. La cité éclipse dans les relations internationales ses voisines. La position de Nagar est néanmoins fragile car elle est à la merci de cités plus importantes de la Mésopotamie, comme en particulier Mari. Nagar est devenue une cité tributaire de Mari au même titre qu'Ebla. La déesse de Nagar (Bēlet Nagar) était présente à Mari avant et après la destruction d'Ebla peut-être comme garante de la fidélité de l'alliance de Nagar. À partir du roi de Mari, Enna-Dagan, cette suprématie est contestée. Nagar et Ebla sont devenues des alliées de circonstance. Une telle alliance était indispensable pour Ebla, car Nagar constituait une étape entre la Syrie et le pays de Kiš ou celui de Hamazi. De vastes alliances se forment; elles entraînent le déploiement des armées et une série de conflits qui conduisent à la destruction d'Ebla, de Mari et Nagar. Kiš est absorbée par une nouvelle force : celle de la ville 
d'Akkad dirigée par le roi Sargon. Il peut avoir été l'auteur de la destruction d'Ebla et de Mari quoi que cette question soit débattue. Nagar et son satellite Nabada sont également détruits à cette période, mais si l'auteur en était Sargon on s'attendrait à ce qu'il s'en vante dans une de ses inscriptions. Quel que soit l'ennemi qui y a mis le feu, cette issue dramatique a marqué un tournant dans l'histoire de la Haute Djéziré qui ne retrouva pas ultérieurement le même niveau de fortune et de rayonnement.

La fin des archives d'Ebla, sans compter celle de Nabada, plonge la Haute Djéziré dans une nouvelle période obscure. Il s'agit d'une obscurité due à la raréfaction des documents et non d'un âge de barbarie, même si un déclin des centres urbains semble s'être produit. Ce sont les fouilles ou surtout les prospections entreprises dans la région qui ont montré une réduction de l'occupation. Mais le phénomène n'est pas homogène. Certains scenarii catastrophiques ont même été envisagés. Pourtant plusieurs grands sites ont continué à s'épanouir dans la zone. La pratique de l'écrit a persisté. Mais aucune découverte épigraphique aussi spectaculaire que celle de Tell Beydar n'a jusqu'à présent été réalisée.

La prise en main de la région par l'empire d'Akkad est surtout effective sous le règne de Narām-Sîn, mais sans doute les Akkadiens y sont-ils déjà implantés sous Rimuš parce qu'une inscription votive qu'on peut lui attribuer a été retrouvée à Nagar. Elle a probablement était offerte à une divinité locale à l'occasion d'une victoire contre l'Élam et le Parahšum. Les Akkadiens font de Nagar leur fief principal dans la région et la ville connaît une renaissance.

Les imposants édifices construits à Nagar comme le «palais fortifié de NarāmSîn » (peut-être seulement un magasin fortifié) témoignent d'une volonté de renforcer l'autorité akkadienne après la phase de la Grande Révolte qui a failli être fatale à l'empire. La tradition épique à l'époque paléo-babylonienne veut que Nagar y ait pris part contre Narām-Sîn sous l'égide d'un roi nommé Ingi. Légendaire ou non on peut admettre que le thème repose sur un souvenir historique réel mais peut-être mal situé chronologiquement à moins qu'il s'agisse d'un amalgame entre plusieurs traditions épiques (souvenir d'une campagne contre Nagar, éventuellement en rapport avec celle bien connue contre la lointaine Apišal?).

Ce contrôle sur le Haut-Habur était cependant partiel. Urkiš, dans la partie nord du Haut-Habur était indépendante sous Narām-Sîn. D'abord combattue par lui-même qui y mena une expédition militaire ou qui du moins la mentionne, dans quelques inscriptions commémoratives, parmi les grandes capitales de l'Ouest qu'il a soumises, elle devint une proche alliée. Vers 2300, un temple fut érigé en son centre sur une plate forme flanquée d'un glacis et d'un escalier monumental. Le sanctuaire devait rester en fonction jusqu'à la fin du deuxième millénaire. La présence hourrite encore discrète avant Sargon est particulièrement marquée à Urkiš, au point que selon son inventeur G. Buccellati, non seulement les scribes locaux auraient adopté les conventions scribales akkadiennes, mais ils auraient adapté également très tôt le cunéiforme à leur langue hourrite. Du moins cette hypothèse repose-t-elle sur une datation haute du premier texte hourrite connu, l'inscription de Tiš-atal. Quoi qu'il en soit l'affirmation d'une entité politique et culturelle singulière face à l'hégémonie d'Akkad se manifeste dans l'usage du titre hourrite endan pour désigner le roi contemporain d'Urkiš. Aux alentours de -2250, Tupkiš qui se présente comme Endan d'Urkiš tira profit de son entente avec Narām-Sîn dont il devint le gendre et érigea un nouveau palais. 
Urkiš était amenée à devenir la principale capitale du Haut-Habur jusqu'à la fin du III ${ }^{e}$ millénaire. Mais comme l'illustre la situation à l'époque amorrite, la vie politique a pu connaître de nombreuses fluctuations. Un document administratif akkadien retrouvé dans les décombres de la « forteresse de Narām-Sîn » de Tell Brak, indique qu'Urgiš a aussi fait partie de la zone d'autorité de Nagar à un certain moment. La juridiction de Nagar s'étendait en outre jusqu'à l'imposante Šehna (Tell Leilan) à l'est du Triangle du Habur. Neuf villes devaient fournir de la main d'œuvre (ĝuruš) à l'administration centrale, pour une raison qui en l'occurrence n'est pas précisée. Nagar, en tête de liste, en fournit le plus grand nombre puis vient Urkiš qui restait donc encore dans l'ombre de sa voisine méridionale. On a remarqué en lisant ce bref document qu'il avait été rédigé en deux fois. Un ajout fut fait in-extremis. Son auteur utilisa une symbolisation des chiffres différente et plus « moderne » pour compter les nouvelles équipes inserrées tant bien que mal d'un côté et de l'autre de l'ancien total qui ne fut pas corrigé. Cela indique l'emploi simultané de deux normes d'écriture ou peut-être même une réforme scribale en cours, ce qui devrait aider à préciser la datation du document. L'équipe des travailleurs de Gagaban (sans doute identique à Kabkab d'après le nom de l'ancien volcan), une cité très proche de Nagar et déjà dans son orbite à l'époque présargonique, était capable de fournir un effectif équivalent à celui d'Urkiš. La région autour de Nagar était donc encore assez prospère.

Mais cette capitale souffrit à l'évidence des vicissitudes de la fin de l'empire d'Akkad. Ses difficultés ont laissé des traces matérielles à l'époque de Šar-kali-šarrī, le successeur de Narām-Sîn. Si une partie de son site continua à être occupée, la ville entra néanmoins dans une phase de lent déclin. Ce phénomène irréversible était dû à l'aridification de la partie méridionale du Triangle du Habur. Nagar a cependant eu l'occasion de récupérer au moins temporairement son indépendance comme le prouve l'existence d'un mystérieux Talpuš-atili (un NP hourrite) « (divin) Soleil du pays de Nagar », fils et par conséquent successeur d'un roi anonyme. Cette inscription trouvée sur une empreinte de sceau a le mérite de montrer qu'une dynastie hourrite s'était installée à Nagar et que, d'une part, elle se situait dans la lignée de Narām-Sîn, le premier roi à s'être fait divinisé de son vivant, tandis que d'autre part, son « idéologie » était commune dans l'espace formant un arc de cercle depuis le piémont de l'Anti-Taurus jusqu'au piémont nord du Zagros lequel était caractérisé par une forte présence hourrite. En effet, un roi de la ville de Kaharar porta à la même période exactement le même titre que Talpuš-atili.

Entre la période de la domination des rois Goutis et celle de la Troisième Dynastie d'Ur, Urkiš s'imposa comme le principal centre politique régional et intégra très probablement dans sa zone d'influence Nagar. Le seul souverain vraiment bien daté de cette période est Annatal grâce à son voyage à Sumer chez Amar-Sîn. Ce succès diplomatique d'Ur fut sans doute préparé bien avant lui par Šulgi puisque les contacts avec Urkiš commencèrent au moins à partir de l'an 47 de Šulgi. Ce rapprochement luimême doit avoir un rapport étroit avec la retentissante défaite subie par le royaume de Mari en l'an 45 de Šulgi (comme l'a montré récemment un document d'Ur III découvert par $\mathrm{P}$. Michalowski) ainsi qu'avec les nombreux problèmes de frontière au nord-est de l'empire d'Ur. 
Si l'on constate que des dynastes hourrites dominaient le Triangle du Habur, la fin du troisième millénaire fut aussi l'époque des « invasions amorrites ». Il est probable que le déclin de la zone méridionale du Haut-Habur s'accompagna du développement du semi-nomadisme et favorisa l'expansion des tribus pastorales, en majorité sémitophones, du Moyen Euphrate ou des groupes implantés entre le sud-est du Sindjar et la Diyala et qu'affrontèrent les rois d'Ur. De même, la chute de l'empire d'Ur III au début du règne d'Ibbi-Sîn a dû affecter directement Urkiš et les autres cités voisines et avoir un impact négatif sur leur propre économie ce qui les a affaiblies face à la montée de nouvelles forces. Celles-ci ont refaçonné le paysage politique de la région dans les décennies qui suivirent. Mais les documents écrits font cruellement défaut dans cette période de transition de près d'un siècle et demi. La multiplication des villages, en particulier dans la partie orientale du Triangle du Habur, a été révélée par les prospections et l'étude des images satellitaires. La culture matérielle dite « Habur Ware » s'y est épanouie.

Lorsque les sources textuelles éclairent à nouveau la situation historique du Triangle du Habur vers le milieu du XIX ${ }^{\mathrm{e}}$ siècle (il s'agit pour cette période ancienne le plus souvent d'informations indirectes recueillies dans des évocations du passé de la génération suivante), on découvre un espace morcelé politiquement dans lequel Urkiš ne joue plus qu'un rôle secondaire, tandis que Nagar n'est plus un royaume mais un sanctuaire prestigieux dont le rôle politique n'est plus que symbolique. La continuité toponymique avec l'époque d'Akkad est flagrante mais partielle : on a fait remarquer par exemple que la Bidiršum paléo-akkadienne était désormais mentionnée dans une lettre du gouverneur de Nahur. Pourtant une quinzaine de noms de lieux attestés par les archives administratives de Brak et Mozan manquent à l'appel au deuxième millénaire. Les populations hourritophones sont une réalité bien vivante surtout dans les cités du Nord, mais l'ensemble du territoire semble avoir été entièrement tribalisé. Les groupes bédouins-Hana, héritiers des Amorrites, dominent le paysage politique et social et ils ont quasiment imposé leur mode d'organisation et créé de nouveaux établissements. Tout au moins la culture urbaine, qui s'est maintenue à travers les siècles et a continué à donner à la région son identité, a-t-elle un caractère métissé et pourrait à la rigueur être qualifiée d'hourro-amorrite voire sémito-hourrite. Mais déterminer ce qui appartiendrait en propre à la culture hourrite, à part l'onomastique et l'usage de la langue, nous paraît bien difficile à réaliser. Les titres d'endan ou « Soleil de son pays » n'étaient plus en usage.

Dans l'ancienne zone de Nagar et Urkiš, deux centres se détachent : Nahur et Ašnakkum. Malgré l'existence d'une hiérarchie entre les cités, aucune ne l'emportait sur l'autre. Cette division fit le jeu des puissances régionales périphériques comme Ešnunna, Ekallatum (sous le règne de Samsi-Addu) et surtout Mari (jusqu'à sa destruction par les Babyloniens) ou encore de capitales régionales comme Andarig au sud du mont Sindjar.

Nous n'avons trouvé aucune mention absolument certaine de Nahur au troisième millénaire. Elle figure peut-être dans un fragment d'itinéraire d'une campagne attribuée à Narām-Sîn. Proche d'Urkiš, si elle existait déjà, elle ne pouvait qu'être dans son ombre. Le dossier examiné datant du début du règne de Zimrī-Lim laisse entendre que c'était devenu au XIX ${ }^{\mathrm{e}}$ siècle l'un des principaux centres à l'Ouest du 
Wadi Djagdjag. Elle avait été une étape des marchands entre Assur et Kaniš en Anatolie. Après le règne de Samsī-Addu, qui avait muselé et privé de leur indépendance les principautés du Haut-Habur, Nahur fut l'objet d'une intense compétition entre plusieurs acteurs régionaux dans les premières années de Zimrī-Lim. On s'est penché en particulier sur un document qui n'est connu que par une transcription inédite de M. Birot retrouvée il y a peu. Il témoigne d'une nouvelle péripétie de l'éphémère Yaqbi-Addu. Il se trouve que ce prince local assiégea Ašnakkum, voisine de Nahur, avant de s'en rendre maître et de s'en proclamer roi. D'autres documents inédits ont montré qu'au même moment, à quelques kilomètres de là, à Nahur, un énigmatique SamsīAddu avait pris le pouvoir et s'était allié à Yaqbi-Addu. Puis ils avaient entamé une négociation avec Mari et parlementé avec le chef des groupes transhumants, rallié à Zimrī-Lim. Leurs prétentions révèlent une volonté politique de faire du Haut-Habur par le biais d'une union des principales cités de la région une puissance équivalente à celle de Mari. Si cette ambition fut illusoire, elle s'appuyait certainement sur le souvenir d'un passé supposé plus glorieux ce qui n'est pas démenti par le bref panorama historique qui vient d'être brossé. Malgré la rapide défaite du roi de Nahur, la situation resta localement instable ce qui força Zimrī-Lim à annexer la ville dans sa cinquième année de règne. Elle fut désormais privée de roi, mais dotée d'un gouverneur chargé de son redressement. En faisant venir des colons de Mari et des cités voisines de Nahur comme Urkiš pour la repeupler, il eut probablement l'idée de transformer Nahur en un centre confédéral au profit de Mari et des Bédouins-Hana ses fidèles alliés. Parmi les nombreuses délibérations entre gens de Mari et autorités locales que cette entreprise avait suscitée, l'une des plus importantes, eut lieu à Urkiš même, peut-être sur la place au pied du grand temple de la cité que les archéologues ont dégagé et qui conviendrait parfaitement à une assemblée générale. Les difficultés de l'occupation de Nahur sont amplement détaillées et il faut croire que la colonisation n'a pas été une complète réussite car Nahur apparaît surtout réduite à sa fonction de cité garnison. La troupe mariote en poste à cet endroit était chargée de la surveillance des cités voisines et intervenait à tout moment en cas de nécessité pour rétablir l'autorité du suzerain.

Le contrôle du Haut Habur convoité par les États mésopotamiens périphériques s'avéra toujours complexe et incertain. Ce territoire était excentré sur le plan géopolitique tout en étant un lieu de passage obligé entre l'Est et l'Ouest; sa population était mixte, ses institutions fortes et retorses, son espace plutôt homogène et réduit était morcelé en petites cités-États querelleuses, maintenues ensemble par une sorte d'esprit fédéraliste souple. Cette situation s'imposa après la chute de Nagar présargonique mais rien ne prouve que l'emprise de cette dernière se soit étendue à tout le Haut-Habur. Les villes étaient bien plus modestes que leurs homologues du Tigre et de l'Euphrate et aucune d'entre elles n'imposa durablement son autorité sur les autres si bien que le centre de gravité politique, s'il y en eut jamais un seul, s'est constamment déplacé d'un point à l'autre du Triangle du Habur. 